\title{
Cassini Ovals in Dynamic Geometry of Polynomials
}

\author{
Gagik Aghekyan ${ }^{1}$, Karen Sahakyan,"* \\ ${ }^{1}$ Department of Applied Mathematics and Computer Science of Russian-Armenian University, Armenia \\ ${ }^{2}$ Department of Mathematics and Mechanics of Yerevan State University, Armenia
}

Copyright (C) 2015 Horizon Research Publishing All rights reserved.

\begin{abstract}
In this paper, we investigate the behavior of critical points of some polynomials whose roots are the vertices of some parallelogram, in case of rotation two of them on a given circle. In this case, the trajectory is the Cassini ovals.
\end{abstract}

Keywords Critical Points, Geometry of Polynomials

\section{Introduction}

The branch of mathematics, named after book of American mathematician Morris Marden [1] as Geometry of Polynomials, gives a geometric relationship between the zeroes of a polynomial with complex coefficients and the zeroes of its derivative. Marden's theorem [1,2] is the most beautiful theorem in Geometry of Polynomials and conjectures of Sendov [3] and Smale [3,4] are two challenging problems of this branch.

First a dynamic behavior of critical points of some polynomials, when moving one of the roots on the given path, was investigated by the authors in $[5,6]$.

This article is a continuation of research in this area. The main result of which is to prove the following statement:

Theorem 1. If the zeros of fourth-degree polynomials are pairwise symmetric with respect to the origin, then the locus of the critical points of the given polynomial, in motion one of the couples around the origin, are Cassini ovals.

A similar result will be formulated in the case of multiple roots.

\section{Main Results}

Turn to the proof of the main statement of this article.

Theorem 1. If the zeros of fourth-degree polynomials are pairwise symmetric with respect to the origin, then the locus of the critical points of the given polynomial, in motion one of the couples around the origin, are Cassini ovals.

Proof. Without loss of generality assume that

$$
z_{1}=-a, z_{2}=a, z_{3}=b e^{i \varphi}, z_{4}=-b e^{i \varphi}
$$

is items found roots of the next polynomial

$$
P(z)=\left(z^{2}-a^{2}\right)\left(z^{2}-b^{2} e^{2 i \varphi}\right)
$$

With derivative

$$
P^{\prime}(z)=2 z\left(2 z^{2}-a^{2}-b^{2} e^{2 i \varphi}\right)
$$

Let $z=x+i y$ then

$$
\begin{aligned}
x^{2}- & y^{2}+i 2 x y=\frac{a^{2}+b^{2} e^{2 i \varphi}}{2} \\
= & \frac{a^{2}}{2}+\frac{b^{2}}{2} \cos 2 \varphi+i \frac{b^{2}}{2} \sin 2 \varphi
\end{aligned}
$$

and

$$
\begin{gathered}
\left\{\begin{array}{c}
x^{2}-y^{2}=\frac{a^{2}+b^{2} \cos 2 \varphi}{2} \\
x y=\frac{b^{2} \sin 2 \varphi}{4}
\end{array}\right. \\
x^{2} y^{2}=\frac{b^{4}}{4} \times \frac{1-\cos 2 \varphi}{2} \times \frac{1+\cos 2 \varphi}{2} \\
\frac{b^{4}}{16}\left(1-\cos ^{2} 2 \varphi\right)=\frac{b^{4}}{16}\left(1-\left(\frac{2\left(x^{2}-y^{2}\right)-a^{2}}{b^{2}}\right)^{2}\right)
\end{gathered}
$$

Thus

$$
16 x^{2} y^{2}=b^{4}-\left(2\left(x^{2}-y^{2}\right)-a^{2}\right)^{2}
$$

And

$$
16 x^{2} y^{2}+4\left(x^{2}-y^{2}\right)^{2}=b^{4}+4 a^{2}\left(x^{2}-y^{2}\right)-a^{4}
$$

Whence

$$
4\left(x^{2}+y^{2}\right)^{2}=b^{4}+4 a^{2}\left(x^{2}-y^{2}\right)-a^{4}
$$

Then

$$
\left(x^{2}+y^{2}\right)^{2}-a^{2}\left(x^{2}-y^{2}\right)=\left(\frac{b}{\sqrt{2}}\right)^{4}-\left(\frac{a}{\sqrt{2}}\right)^{4}
$$

Theorem is proved.

Remark 1. If the zeros of fourth-degree polynomials are symmetric with respect to the origin, then the locus of the critical points of the given polynomial, in motion one of the couples around the origin, are lemniscates of Bernoulli. 
Remark 2. If the zeros of fourth-degree polynomials are pairwise symmetric with respect to the origin, then the locus of the critical points of the given polynomial, is the origin and the foci of this midpoint-tangent inellipse whose zeroes are the vertices of the quadrangle.

This assertion follows from Proposition 5 of the article [7]. Similarly, we can prove the following theorem.

Theorem 2. If two pair of zeros of polynomials are pairwise symmetric with respect to the origin and symmetric zeros have the same multiplicity, then the locus of the nontrivial critical points of the given polynomial, in motion one of the couples around the origin, are Cassini ovals.

\section{Conclusions}

In the paper we consider the dynamic behavior of the critical points of some polynomials, with the motion of some of the roots of the polynomial along a given trajectory.

A similar problem was studied in first time in [5] and it is of interest a more general formulation of this problem in the case of polynomials of general form.

\section{Appendix}
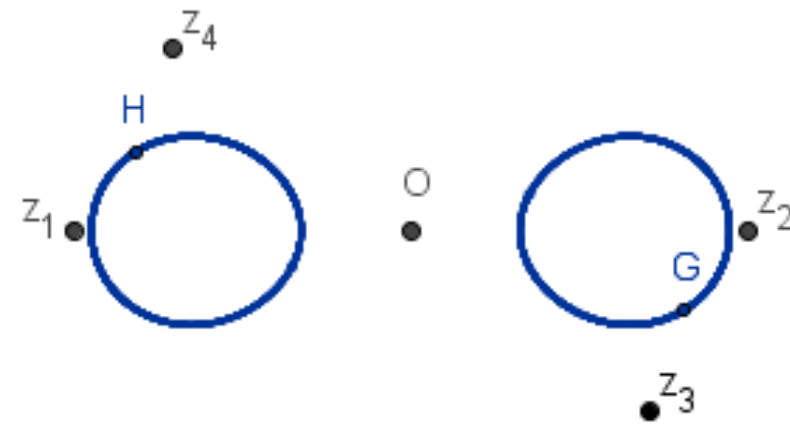

Figure 1. Cassini oval in case of $b<a$.

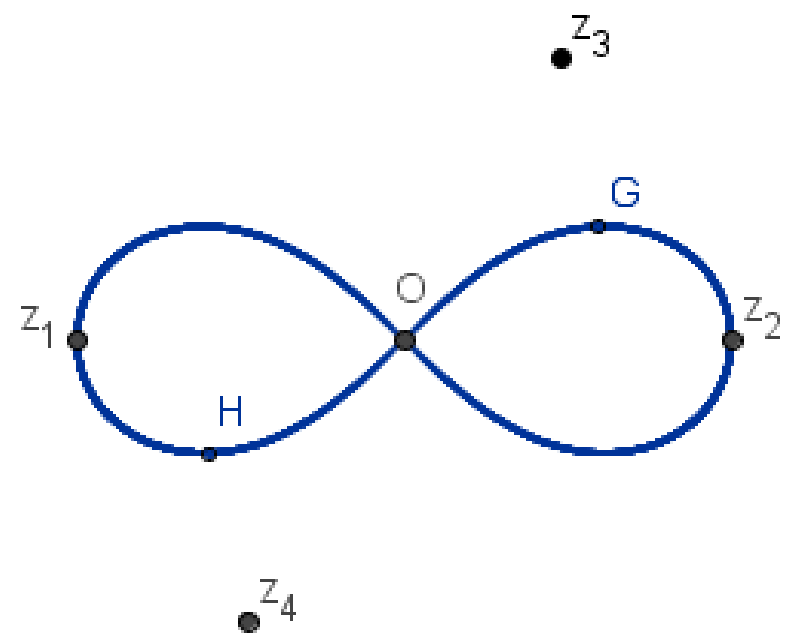

Figure 2. Cassini oval in case of $b=a$.

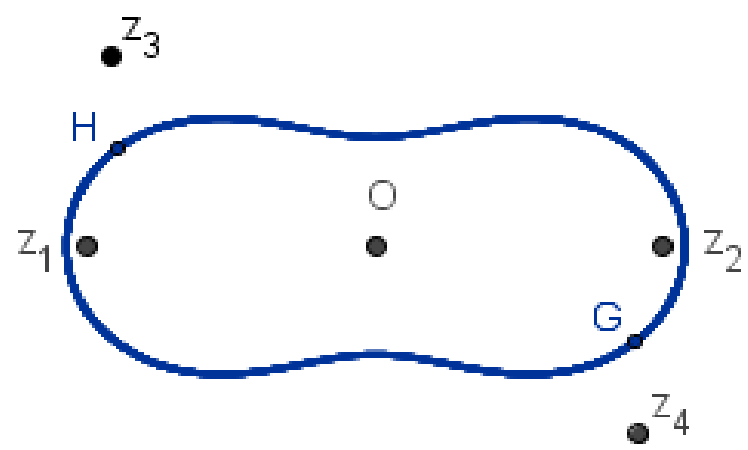

Figure 3. Cassini oval in case of $b>a$. 


\section{REFERENCES}

[1] M. Marden, Geometry of Polynomials, AMS, 1966.

[2] D. Kalman, An Elementary Proof of Marden's theorem, The Amer. Math. Monthly, Vol.115 (2008) pp.330-338.

[3] G. Schmeisser, The conjectures of Sendov and Smale, Approximation Theory (a volume dedicated to Blagovest

Sendov), Sofia Darba, 2002, pp 353-369.

[4] S. Smale, The fundamental theorem of algebra and complexity theory, Bulletin of AMS 4(1981) pp.283-304.

[5] G. Aghekyan, K. Sahakyan, Dynamic Geometry of Cubic Polynomial, Universal Journal of Applied Mathematics Vol. 1 No 4(2013) pp. 207-211

[6] G. Aghekyan, K. Sahakyan, On critical points of some polynomials, Proceedings of the YSU, No 2(2013) pp.64-66

[7] J.Parish, On the derivative of a vertex polynomial, Forum Geometricorum Vol.6 (2006) pp. 285-288. 